\title{
Management of Anesthesia for Rubinstein-Taybi syndrome
}

\author{
Cheon-Hee Park, Kyung Hee Park, and Bo-Yoon Choi \\ Department of Anesthesia and Pain Medicine, Gwangju Christian Hospital, Gwangju, Korea
}

Rubinstein-Taybi syndrome (RTS) is a chromosomal mutation disease, which was first described by Rubinstein and Taybi in 1963. Respiratory infection such as aspiration pneumonia and congenital heart disease are leading causes of death during the one-year post-natal period. The patients with the diseases have difficulties in receiving intubations due to the craniofacial abnormality and anesthesia due to respiratory infection and arrhythmia [1].

A 10-year-old female patient weighing $40 \mathrm{~kg}$ (75-90 percentile) with a height of $135 \mathrm{~cm}$ (25-50 percentile) had RTS with a microdeletion of chromosome 16 , and had no particular medications. She was classified with a grade 1 mental retardation. She usually suffered from sleep apnea syndrome, and visited our hospital for tonsillectomy (grade III) and adenoidectomy. She received surgery to correct the strabismus under general anesthesia at another hospital two years ago, but she was reportedly discharged without any side effects.

Microcephaly, down slanting palpebral fissures and beaked nose were observed as RTS traits. Moreover, microstomia, macroglossia, narrow palate, micrognathia, and retrognathia were presented. Any abnormal findings were not present on the chest X-ray, electrocardiogram, and echocardiogram, but difficulties in managing her airways were expected since she was classified as Mallampati class III.

She had been deprived of food for eight hours and was intramuscularly injected with $10 \mathrm{mg}$ of famotidine for the risk of aspiration, and then was transferred to the operating room. However, she was extremely anxious; thus, she was administered ketamine (15 mg) and midazolam (1.5 mg) via IV injection under oxygen saturation monitoring, and was transferred to the operating room.
She was attached to monitoring equipment such as an electrocardiogram, an oxygen saturation monitor, and a noninvasive blood pressure monitoring machine upon arrival at the operating room. Before induction, her vital signs were 120/74 mmHg (BP), 106 beats/min (HR), 20 times/min (RR), and 94\% (SpO2). Oxygen ( $5 \mathrm{~L} / \mathrm{min}$ ) was provided to the patient via a mask, and the ProSeal LMA was prepared.

$70 \mathrm{mg}$ of propofol was administered via IV injection, and 25 mg of rocuronium was intravenously injected after confirming mask ventilation. One hundred seconds later, we performed an intubation with a cuffed tube (reinforced $5.5 \mathrm{~mm}$ ). The laryngoscopic view was defined as Cormark \& Lehane grade II. However, the tube did not advance further around the subglottic area; therefore, intubation was performed with the cuffed tube (reinforced $5.0 \mathrm{~mm}$ ). At that time, vital signs were stable. We began operating by maintaining oxygen $(1 \mathrm{~L} / \mathrm{min})$, air (2 L/min), and sevoflurane (1.5-2.5 vol\%).

Premature ventricular contraction was detected on the EKG three times in a row immediately after the surgery. Premature ventricular contraction continued for five minutes based on the 10 to 15 out of 110 beats $/ \mathrm{min}$. Therefore, we discontinued the surgery and waited, but it recovered to a normal rhythm without any other medication. After that, we resumed the operation. The vital signs during the surgery were $100-120 / 60-80 \mathrm{mmHg}$ (BP), $90-120$ beats/min (HR), $14-16$ times/min (RR), and $99 \%$ (SpO2). We confirmed that self-respiration fully recovered by the time the surgery was completed, and then, glycopyrrolate $(0.2 \mathrm{mg})$ and pyridostigmine $(5 \mathrm{mg})$ were administered. As a result, we transferred the patient to the recovery room.

The patient was extubated as she began opening her eyes and attempted extubation by herself. $2 \mathrm{~L} / \mathrm{min}$ of oxygen was

Corresponding author: Cheon-Hee Park, M.D., Department of Anesthesia and Pain Medicine, Gwangju Christian Hospital, 37, Yangnim-ro, Nam-gu, Gwangju 503-715, Korea. Tel: 82-62-650-5154, Fax: 82-62-650-5116, E-mail: pch1962@hanmail.net

(c) This is an open-access article distributed under the terms of the Creative Commons Attribution Non-Commercial License (http:// creativecommons.org/licenses/by-nc/3.0/), which permits unrestricted non-commercial use, distribution, and reproduction in any medium, provided the original work is properly cited. 
injected through the nasal cannula after confirming her selfrespiration (99\% SpO2). No complications were found after transferring her to the ward; thus, she was discharged two days after the operation.

There are characteristics associated with RTS such as microcephaly, beaked nose, and low-set ears, down slanting palpebral fissures, broad nasal bridge, strabismus, high arched eyebrows, high arched and narrow palate, hypoplastic maxilla, micrognathia, microstomia, and so on. In addition, the signs of RTS include broad thumbs and hallux, polydactyly and syndactyly, short height (male-153 cm, female-147 cm), mental retardation (IQ 25-79, mean 36-51), congenital heart disease such as ventricular septal defect, patent ductus arteriosus, atrial septal defect, coarctation of the aorta, pulmonic stenosis, bicuspid aortic valve, hypertrichosis, cryptorchism, glaucoma, malignant tumor, etc [2].

Congenital tracheal stenosis, abnormal pulmonary lobulation, excess mucous secretion, and gastroesophageal reflux all could increase the risk of aspiration. Some have choanal atresia or nasal septal deviation, so nasal intubation should be evaluated prior to performing the operation. If a brief surgery or ophthalmic surgery is scheduled, LMA could be utilized. It is possible with ProSeal LMA to achieve intermittent positive ventilation. Thus, it reduces the risk of aspiration of the stomach contents and sealing the larynx $50 \%$ more than that of classic LMA [1].

Succinylcholine is a priority option for patients having difficulty in manipulating the airway; however, a 14-year-old patient experienced arrhythmia 15 seconds after an injection of succinylcholine, which was provided by Stirt. After that, multifocal premature ventricular contraction followed by superventricular tachycardia occurred, and then occasional premature atrial contraction was observed. Moreover, the same patient reportedly experienced arrhythmia after receiving neostigmine and atropine two years ago. As a result, we stress the fact that we need to pay attention to the effect of neuromuscular blocking agents on the heart [3]. Injections of neostigmine and atropine, which alter the sympathetic and parasympathetic systems, cause premature atrial or ventricular contractions on average in $42 \%$ of adults. However, it frequently causes arrhythmia more in RTS patients than in people without the disease.

Therefore, sedatives and opioids lead to increasing apnea. Consequently, they must be used carefully. We are able to induce caudal block combined with general anesthesia for infants with RTS who are scheduled for surgery with an incarcerated inguinal hernia. By doing so, opioids and muscle relaxants can be avoided, reportedly resulting in decreased risk of post-operative hypopnea and apnea [4].

RTS patients are expected to experience difficult airway management due to abnormal anatomical structures, and pretreatments will be inevitable after identifying the response to the anesthetics, which is in all likelihood an arrhythmia. In addition, effort to prevent respiratory complications should be made. In this regard, we report this case with a literature review.

\section{References}

1. Twigg SJ, Cook TM. Anaesthesia in an adult with Rubinstein-Taybi syndrome using the ProSeal laryngeal mask airway. Br J Anaesth 2002; 89: 786-7.

2. Stevens CA. Rubinstein-Taybi syndrome. Gene Reviews [serial on the Internet]. 2009 Aug [2009 Aug 20]. Available from http://www. ncbi.nlm.nih.gov/books/NBK1526/.

3. Stirt JA. Succinylcholine in Rubinstein-Taybi syndrome. Anesthesiology 1982; 57: 429.

4. Critchley LA, Gin T, Stuart JC. Anaesthesia in an infant with Rubinstein-Taybi Syndrome. Anaesthesia 1995; 50: 37-8. 